
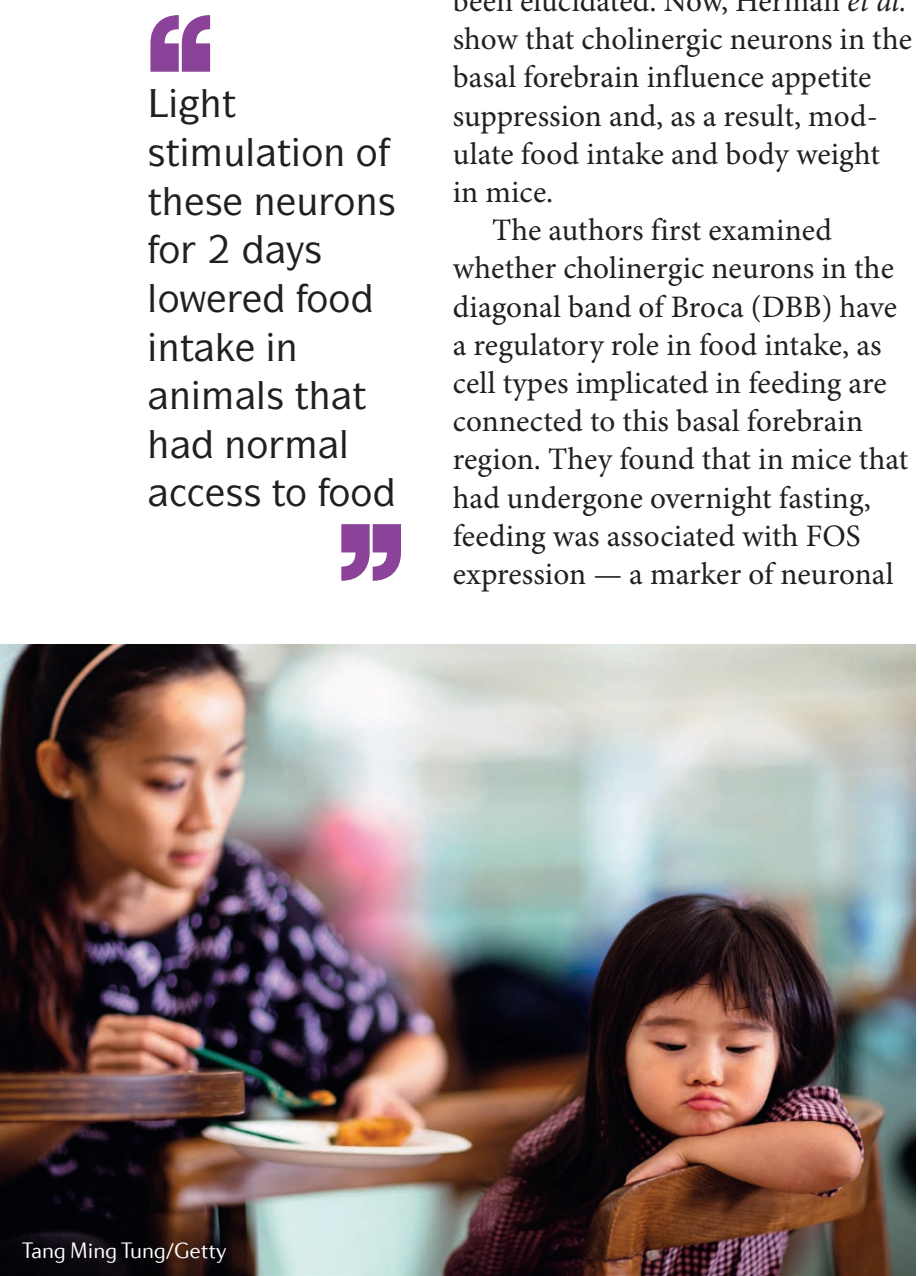

Although acetylcholine has been hypothesized to regulate feeding, the underlying mechanisms have not been elucidated. Now, Herman et al. show that cholinergic neurons in the basal forebrain influence appetite suppression and, as a result, modulate food intake and body weight in mice.

The authors first examined whether cholinergic neurons in the diagonal band of Broca (DBB) have a regulatory role in food intake, as cell types implicated in feeding are connected to this basal forebrain region. They found that in mice that had undergone overnight fasting, feeding was associated with FOS expression - a marker of neuronal

NEURAL CIRCUITS

\title{
Halting hunger
}

activation - in DBB cholinergic neurons. Moreover, they showed that targeted genetic ablation of DBB cholinergic neurons or conditional knockout of choline acetyltransferase in the DBB (which blocked cholinergic transmission) promoted feeding and was associated with obesity in mice. Together, these findings suggest that cholinergic DBB neurons influence feeding.

Next, the authors expressed channelrhodopsin 2 in mouse DBB cholinergic neurons to directly assess, through an optogenetic approach, how activity in these cells affects feeding. Light stimulation of these neurons for 2 days lowered food intake in animals that had normal access to food. A similar effect was observed in animals that underwent overnight fasting and photostimulation for 2 hours in the presence of food. Thus, these data indicate that activation of DBB cholinergic neurons inhibits feeding.

What are the targets of DBB cholinergic neurons? Conditional expression of a presynaptic terminal marker in these cells revealed that they form synapses in various brain regions, including the hypothalamus. Interestingly, the arcuate nucleus of the hypothalamus contains neuronal populations that are known to regulate food intake, including pro-opiomelanocortin-expressing neurons (POMC neurons), the activity of which promotes satiety. Mice in which the DBB was ablated showed a reduction in the level of Pomc transcripts in this nucleus, suggesting that $\mathrm{DBB}$ cholinergic neurons might regulate POMC neuron-mediated satiety signalling.

A previous study showed that activation of acetylcholine receptors on POMC neurons suppressed appetite, and another study revealed connectivity between $\mathrm{DBB}$ neurons and POMC neurons. Here, through the use of virus-mediated retrograde tracing, the authors found that both cholinergic and non-cholinergic DBB neurons project to the hypothalamus. Moreover, in fasted animals presented with food, optogenetic activation of the termini of DBB cholinergic neurons in the ventral hypothalamus suppressed food intake, and this effect could be partially blocked by administration of a nicotinic acetylcholine receptor antagonist. Taken together, these data indicate that DBB cholinergic neurons modulate food intake by activating hypothalamic neurons that suppress appetite.

Darran Yates

ORIGINAL ARTICLE Herman, A. M. et al.

A cholinergic basal forebrain feeding circuit modulates appetite suppression. Nature $\mathbf{5 3 8}$, 253-256 (2016) 